\title{
Multifunctional Branched Nanostraws-Electroporation Platform for Intracellular Regulation and Monitoring of Circulating Tumor Cells
}

Authors: Gen $\mathrm{He}^{\mathrm{a}}$, Jianming Feng ${ }^{\mathrm{a}}$, Aihua Zhanga, Lingfei Zhou ${ }^{\mathrm{a}}$, Rui Wen , Jiangming $\mathrm{Wu}^{\mathrm{a}}$, Chengduan Yang ${ }^{\mathrm{a}}$, Jiang Yang ${ }^{\mathrm{b}}$, Chunwei $\mathrm{Li}^{\mathrm{a}}$, Demeng Chen ${ }^{\mathrm{a}}$, Ji Wang ${ }^{\mathrm{a}}$, Ning $\mathrm{Hu}^{\mathrm{a}}$, and Xi Xie ${ }^{\mathrm{a}, *}$

\section{Affiliations:}

${ }^{a}$ The First Affiliated Hospital of Sun Yat-Sen University; State Key Laboratory of Optoelectronic Materials and Technologies, School of Electronics and Information Technology, Sun Yat-Sen University, Guangzhou, 510006, China

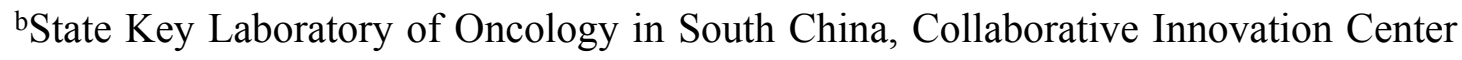
for Cancer Medicine, Sun Yat-sen University Cancer Center, Guangzhou 510060, China.

*To whom correspondence may be addressed. Corresponding to: $\mathrm{Xi} \mathrm{Xie}$, xiexi27@mail.sysu.edu.cn 


\section{Experimental methods}

Fabrication of BNS array: The nanostraws were fabricated using purchased tracketched polycarbonate membrane (GVS) with pores of $400 \mathrm{~nm}$ in diameter and pore densities of $2 \times 10^{8}$ pores $/ \mathrm{cm}^{2}$ as the template. A two-step of sequential atomic layer deposition (ALD) of $40 \mathrm{~nm}$-thick $\mathrm{ZnO}$ and $10 \mathrm{~nm}$-thick $\mathrm{Al}_{2} \mathrm{O}_{3}$ layer was operated after the membranes were placed in the chamber of ALD system (Syskey Technology), resulting in that all the surfaces of the membrane were coated with $\mathrm{ZnO} / \mathrm{Al}_{2} \mathrm{O}_{3}$ hybrid layer. After deposition, the $\mathrm{ZnO} / \mathrm{Al}_{2} \mathrm{O}_{3}$ layer on the top surface was completely removed by mechanical polishing, followed by an oxygen plasma etching process at 80 $\mathrm{W}$ and $100 \mathrm{~Pa}$ for 20 min to remove the exposed polymer and produce the nanostraws array. Subsequently, the nanostraws membrane was subjected to a hydrothermal growth of $\mathrm{ZnO}$ nanowires in the aqueous solutions containing $25 \mathrm{mM} \mathrm{Zn}\left(\mathrm{NO}_{3}\right)_{2}$ and $25 \mathrm{mM}$ hexamethylenetetramine at $90{ }^{\circ} \mathrm{C}$ for $1 \mathrm{~h}$, preparing the nanobranches on the surface of nanostraws. Finally, $\sim 10 \mathrm{~nm}$-thick $\mathrm{Al}_{2} \mathrm{O}_{3}$ layer was sputtered on the surface of the branched nanostraws array. SEM characterization of the PC membrane before ALD, after $\mathrm{ALD}$ of $\mathrm{ZnO}, \mathrm{Al}_{2} \mathrm{O}_{3}$, and after mechanical polishing were shown in Supplementary Figure 1.

Branched nanostraws-electroporation set-up: The BNS membrane was integrated into a microfluidic device by assembling it between a polydimethylsiloxane (PDMS) bottom layer with a microfluidic channel and an upper PDMS chunk with a cell culture well. The microfluidic channel was $\sim 2 \mathrm{~mm}$ in width, $\sim 1.5 \mathrm{~cm}$ in length, and $\sim 200 \mu \mathrm{m}$ in height, serving as a reservoir to load drug or collect the extracted cytosolic contents. An upper PDMS layer with a cut-out hole of $\sim 10 \mathrm{~mm}$ in diameter and $\sim 5 \mathrm{~mm}$ in height acted as a cell culture chamber. Before device packaging, the BNS substrate and PDMS modules were treated by $\mathrm{O}_{2}$ plasma at $100 \mathrm{~W}$ for $20 \mathrm{~s}$ to clean the surfaces. Then the bottom PDMS layer was stuck on the top of indium tin oxide (ITO)-coated glass by non-crosslinked PDMS. The BNS membrane and upper PDMS chunk were successively adhered over the top surface. In the final packaging step, the device was cured at $80{ }^{\circ} \mathrm{C}$ for $4 \mathrm{~h}$ to glue the PDMS. Before modifying specific antibodies on the 
surface of BNS, the devices were sterilized with UV light for $30 \mathrm{~min}$.

Surface modification of specific antibodies: The protocol for anti-EpCAM modification was similar to our previous work, as depicted in Supplementary Figure 3. The substrates were soaked in 4\% (v/v) 3-mercaptopropyl trimethoxysilane (MPTMS) in ethanol for $12 \mathrm{~h}$. After a gentle washing with ethanol, the substrates were reacted with GMBS solution (GMBS was dissolved in DMSO and then diluted to $250 \mu \mathrm{M}$ by ethanol) for $60 \mathrm{~min}$. Next, $10 \mu \mathrm{g} / \mathrm{mL}$ of streptavidin (SA) was covered onto the GMBScoated substrates at room temperature for $60 \mathrm{~min}$, followed by rinsing with PBS more than 3 times. Finally, the SA-functionalized substrates were immersed in $50 \mu \mathrm{L}$ biotinylated anti-EpCAM solution $(10 \mu \mathrm{g} / \mathrm{mL}$ in PBS $)$ for $30 \mathrm{~min}$.

Cancer cells capture: The protocol for cancer cell capture was similar to our previous work $^{1}$. After fabrication of the microfluidic devices, $400 \mu \mathrm{L}$ MCF7 cell suspensions $\left(\sim 10^{6}\right.$ cells $/ \mathrm{mL}$ ) in cell medium (containing 90\% DMEM (Gibco), 9\% fetal bovine serum (Gibco) and 1\% antibiotics) were seeded into the upper culture well and then cultured at $37{ }^{\circ} \mathrm{C}$ and $5 \% \mathrm{CO}_{2}$ in the cell incubator. After incubation, the substrates were gently washed with the medium at least 5 times. Before cell enumeration, the nucleus was stained with Hoechst 33342 for $10 \mathrm{~min}$. Then, at least ten fluorescence images were randomly recorded on the different locations of substrates to count the captured cells using fluorescence microscopy. The capture efficiency was defined as the ratio of the number of the captured cells on the substrates and total cell number in $400 \mu \mathrm{L}$ MCF7 cell suspensions. In every capture experiment, at least five independent devices were measured in parallel to generate an error bar for the cell capture efficiency.

Cell capture assay for artificial CTCs blood samples: Different number of MCF7 cells $(10,20,50,100$, and 250 cells) were added into $1 \mathrm{~mL}$ whole blood samples taken from healthy human, to form the artificial CTCs samples. The MCF7 cells were prestained with CellTracker Green (Invitrogen). Then $1 \mathrm{~mL}$ CTCs samples were seeded into the culture wells of the anti-EpCAM-coated BNS-integrated microfluidic devices and incubated for $60 \mathrm{~min}$. After capturing, the wells were gently rinsed with medium for three times and imaged via fluorescence microscope, followed by counting the 
numbers of captured MCF7 cells. After that, the cell nuclei were stained with Hoechst 33342 to obtain the number of WBCs non-specifically adhered on the BNS. The capture purity was obtained by dividing the quantity of MCF7 cells by the total cells number adhered on the anti-EpCAM-coated BNS. The three-color immunocytochemistry was utilized to identify MCF7 cells. After $60 \mathrm{~min}$ of cell capture, the cells were fixed with 4\% paraformaldehyde in PBS for $10 \mathrm{~min}$. Following that, $50 \mu \mathrm{L}$ of Alexa Fluor 568labeled anti-CK19 and Alexa Fluor 488-labeled anti-CD45 were sequentially added into the wells for 30 min, respectively. The cell nucleus was stained with Hoechst 33342 for nucleus staining. Finally, the cells were observed using fluorescence microscope.

SEM characterization of cell-substrate interfaces: After MCF7 cells were captured on the substrates, the samples were fixed in $2.5 \%$ glutaraldehyde solution for $4 \mathrm{~h}$. Then the cells were sequentially treated by ethanol aqueous solution with increasing concentrations $(30 \%, 50 \%, 70 \%, 90 \%, 100 \%)$. Immediately, a critical point drying (CPD) process was conducted on the samples for dehydration, followed by coated with $\mathrm{Au}$ for SEM characterization.

Simulation of BNS-electroporation: The electric field and electric potential distribution through the BNS were stimulated using the AC/DC Module (steady state) of the COMSOL Multiphysics finite-element-analysis software (COMSOL Inc). Both 3D model and 2D model were constructed and calculated, respectively. The modeling and simulation were detailed in Supplementary Figure 8.

Intracellular delivery by BNS-electroporation: For PI delivery, MCF7 cells experienced a further culture for $24 \mathrm{~h}$ after captured by the BNS. Pt wire was placed in cell medium in the upper cell chamber and served as a negative electrode, while the underneath ITO-coated conducting glass as a positive electrode. After $0.1 \mathrm{mg} / \mathrm{mL}$ PI dye was loaded into the underlying fluidic channel, a cluster of transient electric pulses of $15 \mathrm{~V}$, with $200 \mu$ s duration time, frequency of $10 \mathrm{~Hz}$, and total pulse period of $60 \mathrm{~s}$ (denoted as $15 \mathrm{~V} / 200 \mu \mathrm{s} / 10 \mathrm{~Hz} / 60 \mathrm{~s}$ ) were applied between the ITO glass and Pt electrodes, followed by another 5-min incubation to allow PI dye to enter the cells. Subsequently, the top cell culture well and the underneath fluidic channel were both 
washed three times and replaced with fresh medium to reduce the fluorescence background. Hoechst 33342 (blue fluorescence) was used to stain the cell nucleus to identify the cancer cells, and Calcein-AM (green fluorescence) was to confirm that the cells were still alive.

For DNA transfection, Pt wire served as a positive electrode, while the underneath ITO-coated conducting glass as a negative electrode. Similar to the PI experiment, after filling $0.2 \mu \mathrm{g} / \mathrm{uL}$ of pGFP solution into the fluidic channel, electrical pulses $(15 \mathrm{~V} / 200$ $\mu \mathrm{s} / 10 \mathrm{~Hz} / 60 \mathrm{~s}$ ) were applied, followed by an additional culture for 30 minutes to allow the plasmids to enter the cells. After removing the underneath solution and replacing with new medium, the cells were subsequently incubated for $24 \sim 48 \mathrm{~h}$ to complete the protein expression.

Cell viability assays: The viability of the cancer cells before and after captured by BNS were determined via a standard live/dead cell staining assay with calcein-AM/PI dye. For investigating the cell survival after several cycles of repeated electroporation over time, after filling cell medium into the channels, MCF7 cells captured on the four parallel devices were respectively treated with one, two, four and eight cycles of repeated electroporation under $15 \mathrm{~V} / 200 \mu \mathrm{s} / 10 \mathrm{~Hz} / 60 \mathrm{~s}$ pulses, and there existed an incubation period interval of $1 \mathrm{~h}$ between each electroporation operation. The treated devices were further cultured for $24 \mathrm{~h}$ to allow the porated cell membrane to recover, followed by a standard live/dead cell staining procedure with calcein-AM/PI dye to determine the cell viabilities

Caspase-3 extraction assay using BNS-electroporation: Approximately 100,000 $( \pm 2,000)$ MCF7 cells were seeded on the upper chambers of BNS-integrated microfluidic devices and cultured for $24 \mathrm{~h}$. To assure the uniformity and comparability among the experimental group and three other control groups, the number of cells seeded on each device was nearly identical. Prior to electroporation, the caspase- 3 in the blank medium was analyzed and evaluated as control (Control), which was set as the base level (defined as "100\%") for reference to the values in the electroporation experiments. After filling fresh medium into the channels and adding the medium 
containing $1 \mu \mathrm{M}$ staurosporine (STS) into the top wells to induce cell apoptosis of MCF7, we started the repeated electroporation on the devices. At the first sampling point $\left(t_{1}: 0 \mathrm{~h}\right)$, the MCF7 cells were treated with electric pulses of $15 \mathrm{~V} / 200 \mu \mathrm{s} / 10 \mathrm{~Hz} / 60$ $\mathrm{s}$, followed by an additional 30 minutes' incubation to allow the cytosol released from the cells into the underneath channels. Then the underneath medium was pipetted and analyzed with Caspase-3 Colorimetric Assay Kit (BioVision) to quantitatively measure the caspase-3 levels according to the manufacturer's protocol, and the devices were placed back for $3 \mathrm{~h}$ incubation to await the next electroporation. Then in a similar fashion, the devices were repeatedly subjected to the identical procedure at the second $\left(t_{2}: 3 \mathrm{~h}\right)$, third $\left(t_{3}: 6 \mathrm{~h}\right)$ and fourth $\left(t_{4}: 9 \mathrm{~h}\right)$ sampling point, and the caspase-3 levels were quantitatively tested. To rule out the contingency, the extraction assays in the four groups were performed on five parallel microfluidic devices to give the corresponding caspase-3 levels and calculate the error bars.

Analysis of intracellular caspase-3 activity: For analyzing the caspase- 3 in the extracted cytosol, the withdrawn solution in the underneath channels was diluted to 50 $\mu \mathrm{L}$ with fresh medium. The caspase-3 levels were quantified via Caspase-3 Colorimetric Assay Kit (BioVision). In principle, caspase-3 enabled to cleave the substrate acetyl-Asp-Glu-ValAsp (Ac-DEVD) labeled the chromophore $p$-nitroaniline ( $p \mathrm{NA}$ ) (Ac-DEVD- $p \mathrm{NA}$ ) to release $p \mathrm{NA}$. The released $p \mathrm{NA}$ concentrations were calculated based on the absorbance values at $405 \mathrm{~nm}$ and the calibration curve of the defined $p$ NA solutions, giving the activity of caspase-3. According to the manufacturer's protocol, firstly, $50 \mu \mathrm{L}$ of $2 \mathrm{X}$ Reaction Buffer (containing $10 \mathrm{mM}$ DTT) and $5 \mu \mathrm{L}$ of $4 \mathrm{mM}$ DEVD-pNA substrate were added into each extracted sample, followed by incubation at $37^{\circ} \mathrm{C}$ for $2 \mathrm{~h}$. Then the absorbance values at $405 \mathrm{~nm}$ of treated samples were measured in a spectrophotometer. The calibration curve of $p$ NA solutions were obtained by measuring the absorbance of a series of $p \mathrm{NA}$ solutions at the concentration range of 10 to $200 \mu \mathrm{M}$ by diluting the $p$ NA stock solution in $2 \mathrm{X}$ Reaction Buffer.

Cell apoptosis assay by STS treatment: The apoptosis behavior of MCF7 cells under 
$1 \mu \mathrm{M}$ STS treatment was dynamically tracked with CellEvent ${ }^{\mathrm{TM}}$ Caspase-3/7 Green Detection Reagent (Invitrogen). Caspase-3/7 Green Detection Reagent is a novel fluorogenic substrate for activated caspases-3 and 7 and can produce a bright, fluorogenic response with an absorption/emission maximum of $\sim 502 / 530 \mathrm{~nm}$ after activation of caspase-3 or caspase-7 in apoptotic cells. Therefore, the apoptosis behaviors of the captured MCF7 cells were dynamically monitored by tracking the caspase 3/7 activities. During STS treating period, Caspase-3/7 Green Detection Reagent (Invitrogen) solution (in DMSO) was added into the culture medium in the upper wells, forming the final working concentration of $10 \mu \mathrm{M}$. The fluorescence images were recorded at four time points $(0,3,6,9 \mathrm{~h})$ by fluorescence microscope. 


\section{Supplementary figures:}

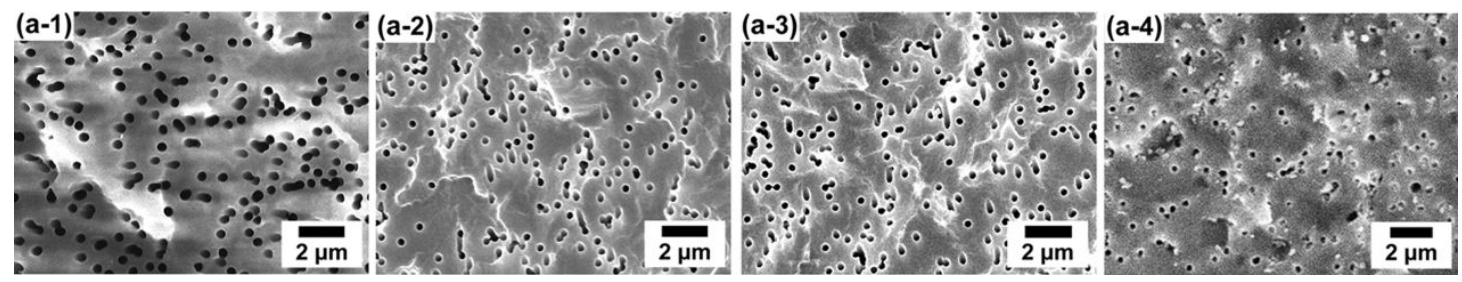

Supplementary Figure 1: SEM characterization of the PC membrane before ALD (a1), after $\mathrm{ALD}$ of $\mathrm{ZnO}(\mathrm{a}-2), \mathrm{ALD}$ of $\mathrm{Al}_{2} \mathrm{O}_{3}$ (a-3), and after mechanical polishing (a-4).

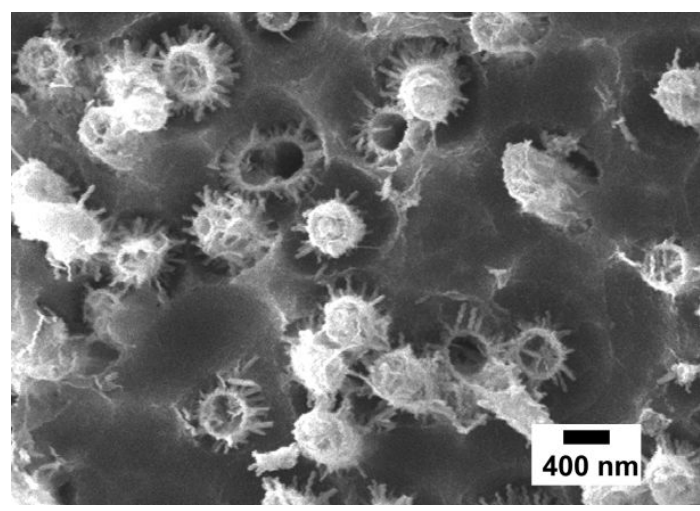

Supplementary Figure 2: SEM characterization of branched nanostraws where the $\mathrm{ZnO}$ nanostraws were prepared without a second ALD of $\mathrm{Al}_{2} \mathrm{O}_{3}$. The SEM image demonstrated that most of the conduits inside the nanostraws were blocked by $\mathrm{ZnO}$ nanowires because the internal sidewall also provided growth sites for $\mathrm{ZnO}$ nanowires.
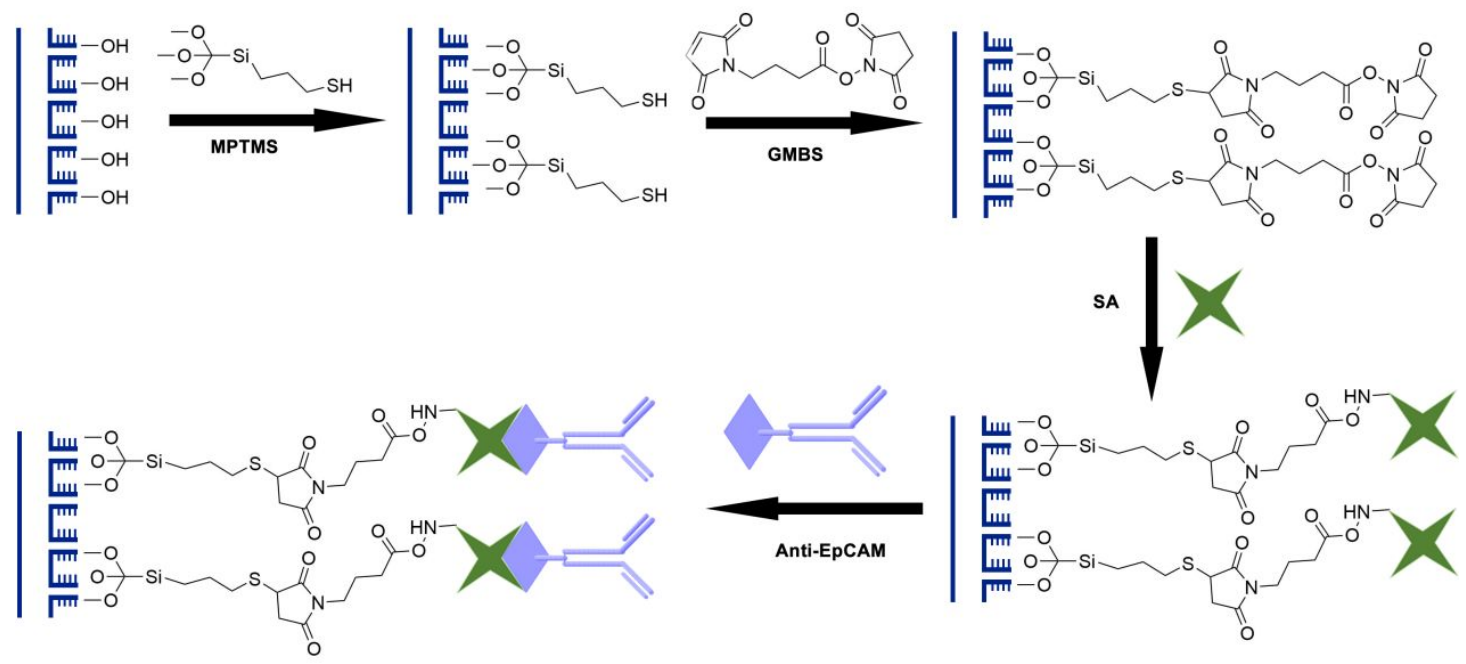

Supplementary Figure 3: Illustration of specific antibody modification onto the surface of BNS. 


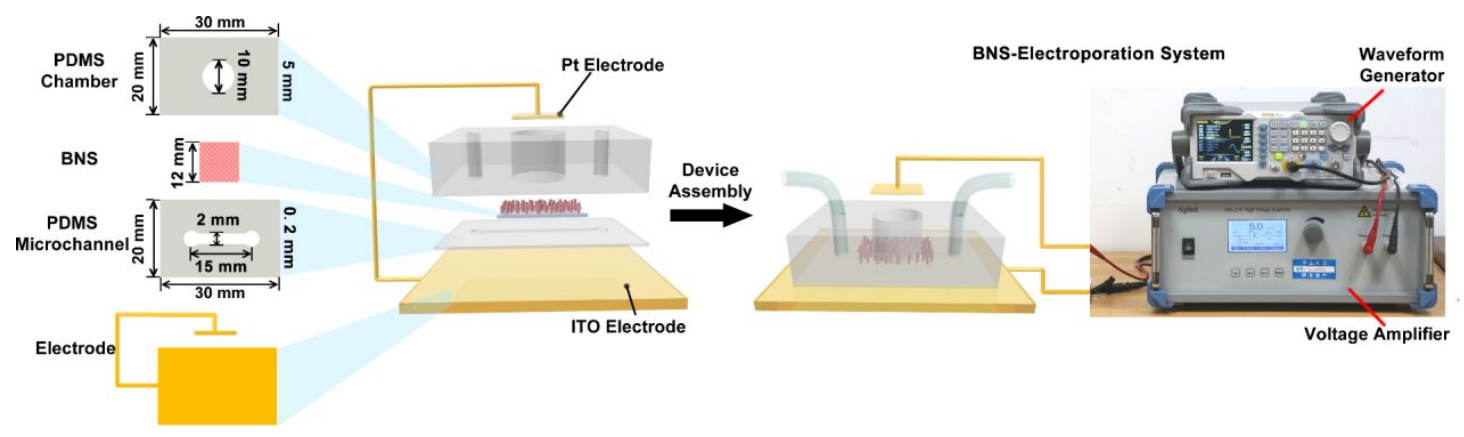

Supplementary Figure 4: The setup of BNS-integrated microfluidic system.
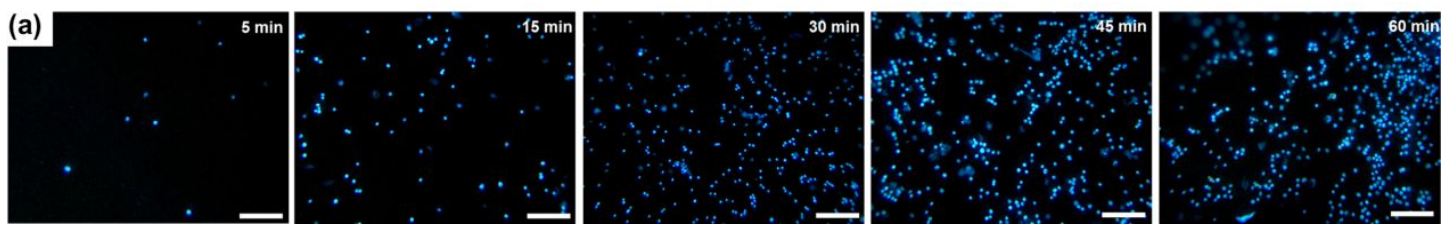

Supplementary Figure 5: A representative group of sequential time snapshots of fluorescence images when the MCF7 cells were captured by the anti-EpCAM-coated BNS. Scale bar: $200 \mu \mathrm{m}$.
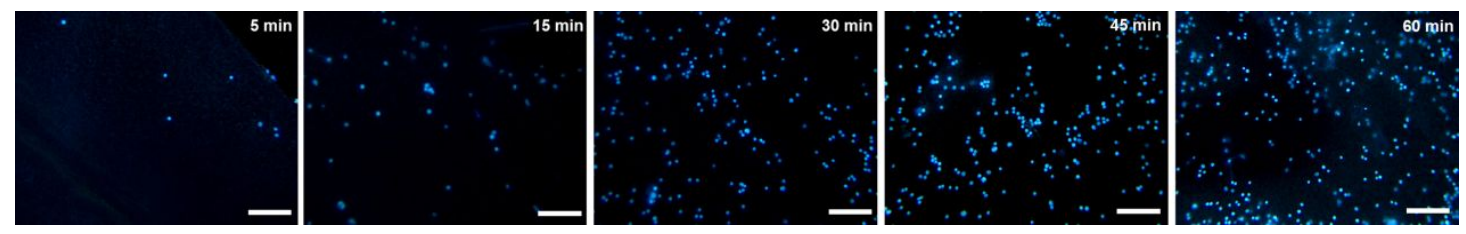

Supplementary Figure 6: A representative group of sequential time snapshots of fluorescence images when the MCF7 cells were captured by the anti-EpCAM-coated NS. Scale bar: $200 \mu \mathrm{m}$.
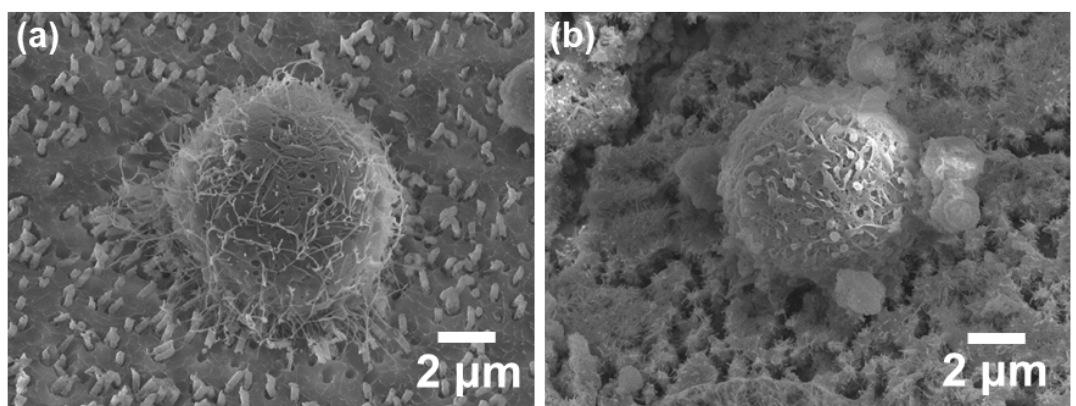

Supplementary Figure 7: SEM images in Figure 2g-h without false color showing the interfaces between the captured MCF7 cells and NS (a), and BNS (b). 

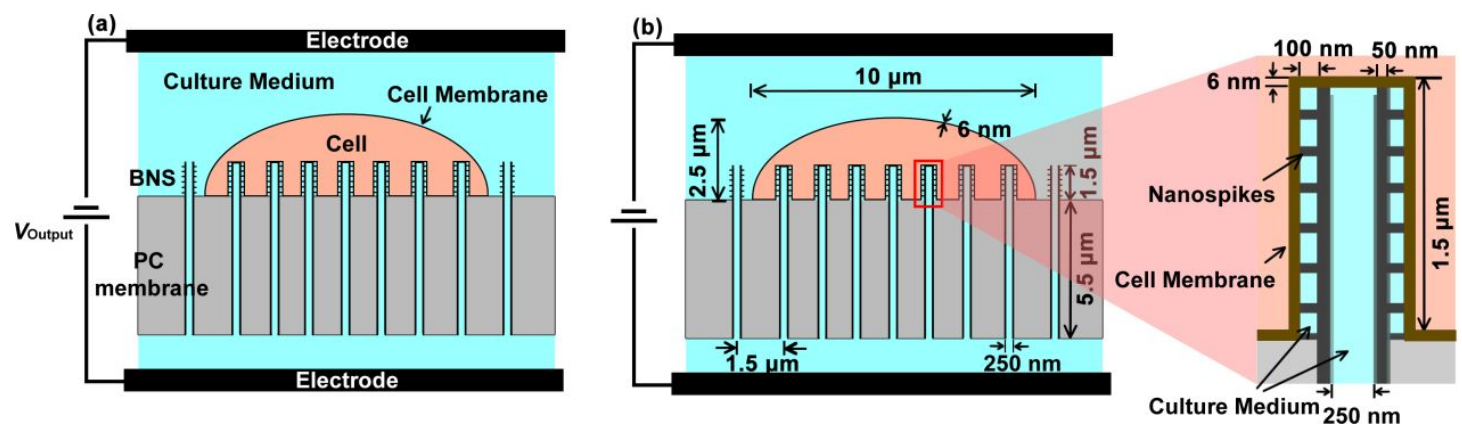

Supplementary Figure 8-1: Schematic of 2D model for calculating the localized electrical field and transmembrane potential on cell membrane by BNS array (a), and the related dimension parameters in simulation (b).
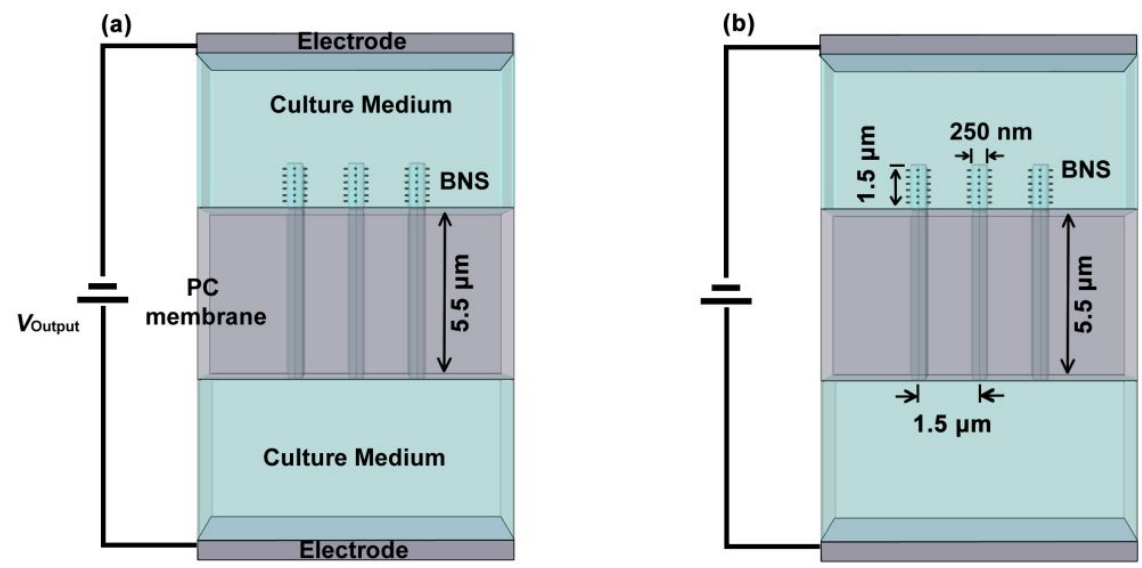

Supplementary Figure 8-2: Schematic of 3D model for simulating the localized electrical potential through the BNS array (a) and the related parameters (b).

Supplementary discussion on theoretical calculation: To quantitatively study the electric field and electric potential distribution through BNS, we simulated the local electric field intensity and electric potential using the AC/DC Module (steady state) of the COMSOL Multiphysics finite-element-analysis software (COMSOL Inc). The fundamental physics equations used in this electrical field modulus includes:

$$
\begin{aligned}
& \mathrm{J}=\sigma \mathrm{E} \\
& \mathrm{E}=\nabla \mathrm{V} \\
& \nabla \cdot \mathrm{J}=0
\end{aligned}
$$

Where $\mathrm{J}$ is the current density, $\mathrm{E}$ is electrical field intensity, $\mathrm{V}$ is electric potential, $\sigma$ is the electric conductivity. 
The Laplace equation is used to calculate the distribution of the electric field and electric potential:

$$
\nabla(\sigma \cdot \nabla \mathrm{V})=0
$$

In our work, for $3 \mathrm{D}$ model, a representative area of $3 \times 3 \mathrm{BNS}$ in array was studied. The BNS uniformly distributed in the PC membrane. The thickness of the PC membrane is $5.5 \mu \mathrm{m}$, and the external diameter of the BNS is $400 \mathrm{~nm}$. The inner diameter of the BNS nano-channel is about $250 \mathrm{~nm}$, and the BNS height is $1.5 \mu \mathrm{m}$. According to the BNS density of $2 \times 10^{8}$ pores $/ \mathrm{cm}^{2}$ in $\mathrm{PC}$ membrane, the center to center distance between two BNS is about $1.5 \mu \mathrm{m}$. The nano-branches are assumed to be 100 $\mathrm{nm}$ in length, and filled with medium on their vacancies. The medium in cell culture reservoir (on the top of the BNS) and the medium in the microfluidic channel underneath the BNS are both modeled as rectangle media. Cell medium was also filled in the nano-channels of each BNS, connecting the medium in the culture well and those in the microfluidic channel. The voltage $(15 \mathrm{~V})$ is applied between the bottom ITO electrode and the top Pt electrode, where the top Pt electrode is set to be grounded. The property of the media (defined by culture medium) is considered homogeneous. In this experiment, the electrical current in the medium relies on the flow of electrolytes. The PC membrane and the oxide materials of BNS are both non-conductive to the electrolyte currents, and thus, the electric field across the PC membrane and the BNS materials can be neglected (grey region in Figure 4a and Supplementary Figure 8). Due to the dielectric constant and conductivity the differences with cell membrane, cytoplasm, BNS and the medium solution, the electric field lines between the two electrodes are mainly distributed in the medium solution. Therefore, only electrical field in the culture medium is considered. Parameters used in the simulation are given in Supplementary Table. 1.

Supplementary Table 1: Parameters of the Three-Layer Model

\begin{tabular}{|c|c|c|}
\hline Symbol & Value & Definition \\
\hline$d$ & $6 \mathrm{~nm}$ & cell membrane thickness \\
\hline$\sigma_{\mathrm{e}}$ & $0.2 \mathrm{~S} / \mathrm{m}$ & electric conductivity of external medium \\
\hline$\sigma_{\mathrm{i}}$ & $0.2 \mathrm{~S} / \mathrm{m}$ & electric conductivity of cytoplasm \\
\hline$\sigma_{\mathrm{m}}$ & $5 \times 10^{-7} \mathrm{~S} / \mathrm{m}$ & electric conductivity of cell membrane \\
\hline
\end{tabular}


"i" is the internal cytoplasm, "e" is the external medium, and " $\mathrm{m}$ " is the cell membrane.

For 2D model, a representative area BNS in array supporting 4 cells was studied. The BNS-device geometry set-up and parameters are the same as those used in the $3 \mathrm{D}$ model. MCF-7 cells are simplified as a semi-ellipsoid with the diameter to be $10 \mu \mathrm{m}$ and the height to $2.5 \mu \mathrm{m}$. The cells are sitting on substrate, while the bottom cell membrane is modeled to be completely deformed around the BNS, in close contact with the BNS opening and the nano-branches. The cell membrane is $6 \mathrm{~nm}$ in thickness, as indicated with the black curves in Figure 4a. The perforation of cell membrane occurs in the order of microseconds as soon as turning on the electric field. We can assume that the external voltage drop between ITO and Pt electrodes is constant in the poration process. The calculated transmembrane potential (the potential difference across the cell membrane) is a fixed value because it reflects the potential difference across the cell membrane when it is breakdown. According to previous experimental and theoretical results, the critical transmembrane potential to porate cell membrane is $0.2 \sim 1 \mathrm{~V} .{ }^{2,3}$ Considering the thickness of cell membrane is $6 \mathrm{~nm}$, the critical electrical filed intensity to cause cell membrane poration is $300 \sim 1600 \mathrm{kV} / \mathrm{cm}$. The existence of the cell changes the distribution of the voltage drop. The transmembrane potential is obtained as:

$$
\Delta \mathrm{V}_{\mathrm{m}}=\mathrm{V}_{\mathrm{m}}\left(\mathrm{S}_{\mathrm{e}}\right)-\mathrm{V}_{\mathrm{m}}\left(\mathrm{S}_{\mathrm{i}}\right)
$$

where $S$ is the surface of cell membrane, "e" indicates "external", and "i" indicates “internal".

The electric field is concentrated around the top ending of BNS, and most of the electric field lines are forced to penetrate through the cell membrane in close contact with the top opening of the BNS. The larger affected cell surface is, the higher cell damage rate will be. The transmembrane potential is highly concentrated at the top of the BNS, while the other cell surface is less affected by the electrical field. The cell membrane proration could highly locally occur around the BNS, without causing cell membrane rupture among other surfaces. 


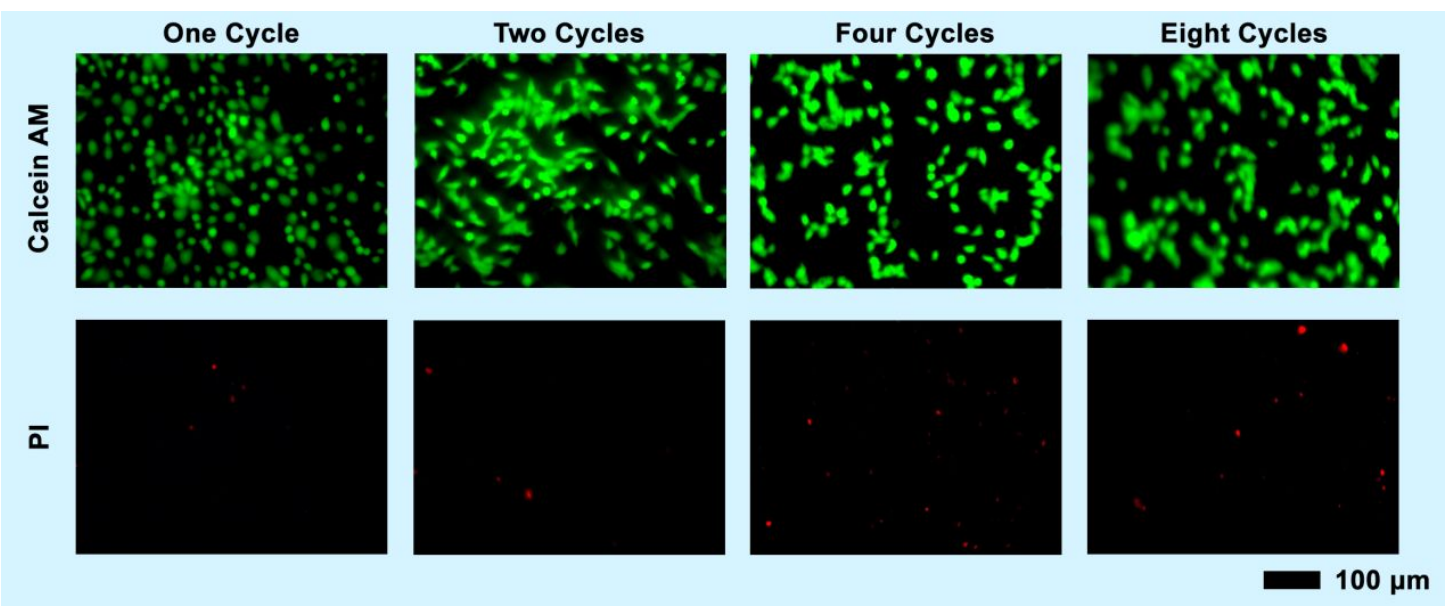

Supplementary Figure 9: The fluorescent images of MCF7 cells in Green and Red channel after subjected to several repeated electroporation operations. The merged images are shown in Figure 5a.

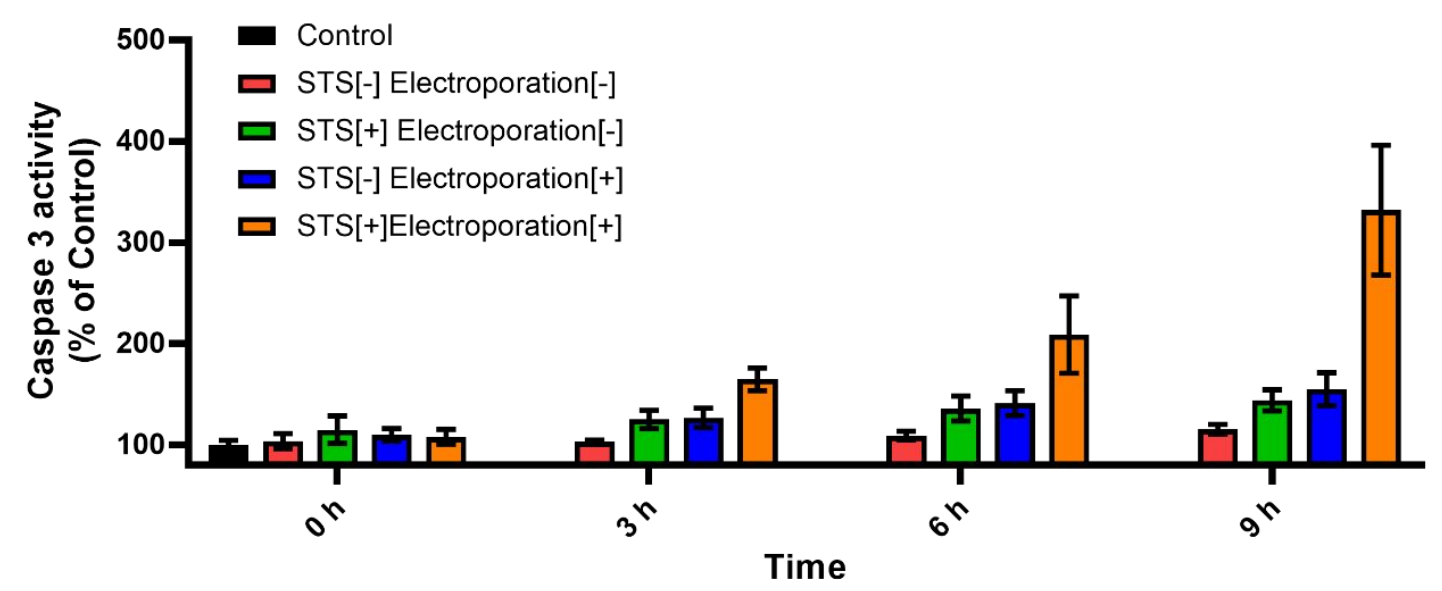

Supplementary Figure 10: Time-course intracellular caspase-3 activity of MCF7 cells at four different group including STS[-]Electroporation[-], STS[+]Electroporation[-], STS[-]Electroporation[+], and STS[+]Electroporation[+]. 

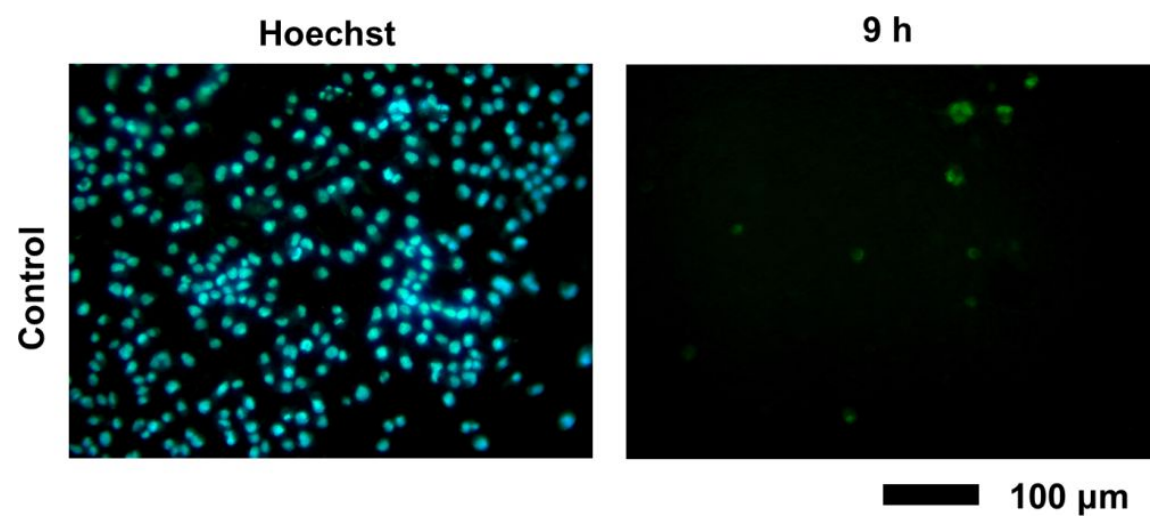

Supplementary Figure 11: The cell apoptosis behavior in the absence of STS after 9 $\mathrm{h}$ incubation as control group.

(a)

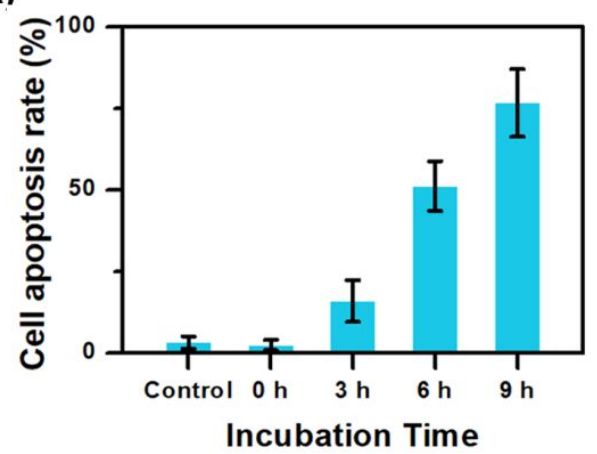

(b)

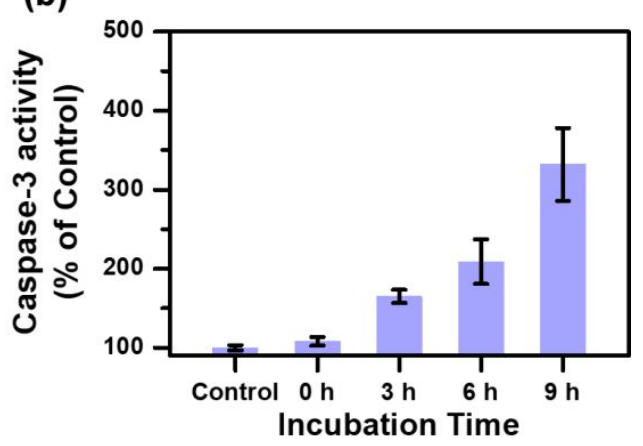

Supplementary Figure 12: (a) Quantitative determination of the cell apoptosis rates under the delivered of $1 \mu \mathrm{M}$ STS by analyzing the caspase 3/7-correlated green fluorescence, and comparison with the time-course trend of intracellular caspase- 3 activity, demonstrating a consistent relationship with incubation time.

\section{Supplementary Figure 13:}

We have performed the cell viability experiments using live/dead cells staining (calcein AM / PI dye) after the caspase-3 extraction assays in the four different groups. The cells were incubated with or without STS for 9 hours, and were treated with or without electroporation (at three time points). The fluorescence images and statistical results of cell viabilities in Supplementary Figure 13 showed that very few cells were stained with red fluorescence of PI dye, indicating the membrane of all the cells were intact and eliminating the possibility of leak of caspase- 3 caused by the dead cells. 

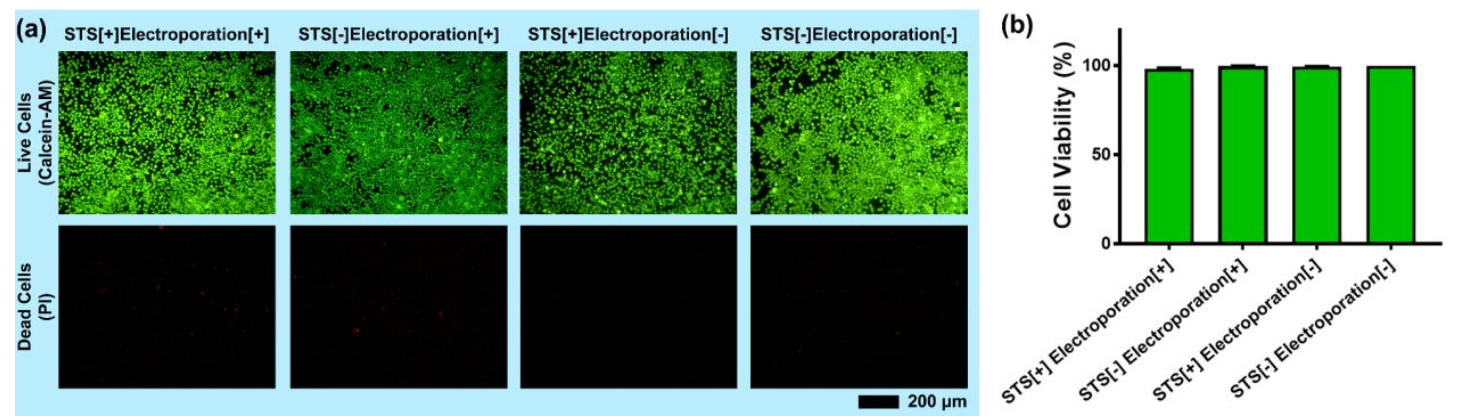

Supplementary Figure 13. (a) Fluorescence images of cell viabilities after caspase-3 extraction assays in four different experimental groups. Green: live cells stained with calcein AM; red: dead cells stained with PI dye. (b) Statistical results of cell viabilities after caspase- 3 extraction assays in four different experimental groups.

\section{References:}

(1) G. He; C. Yang; J. Feng; J. Wu; L. Zhou; R. Wen; S. Huang; Q. Wu; F. Liu; H.-J. Chen; T. Hang; X. Xie. Adv. Funct. Mater. 2019, 29, 1806484.

(2) M. Wang; O. Orwar; J. Olofsson; S. G. Weber. Anal. Bioanal. Chem. 2010, 397, 3235-3248.

(3) G. Zhang; N. Fan; H. Jiang; J. Guo; B. Peng. Journal of Physics: Conference Series 2018, 986, 012018. 